\title{
The use of guidelines for the empirical treatment of hospital-acquired pneumonia
}

\author{
Michael S Miletin MD FRCPC, Charles K Chan MD FRCPC FCCP FACP \\ Division of Respirology, Department of Medicine, The University Health Network \\ and Mount Sinai Hospital, University of Toronto, Toronto, Ontario
}

\begin{abstract}
MS Miletin, CK Chan. The use of guidelines for the empirical treatment of hospital-acquired pneumonia. Can Respir J 2001;8(4):255-260.
\end{abstract}

BACKGROUND: Several practice guidelines for the empirical antimicrobial treatment of hospital-acquired pneumonia (HAP) have been developed, but the acceptance and use of such guidelines are unknown.

OBJECTIVE: To assess physicians' use of empirical HAP guidelines published by the American Thoracic Society (ATS) and by The University Health Network, Toronto, Ontario.

DESIGN: A retrospective assembly and chart review.

SETTING: A university teaching hospital.

PATIENTS: One hundred fifteen consecutive patients who had been diagnosed with pneumonia more than $48 \mathrm{~h}$ after admission to hospital over a 10-month period.

RESULTS: The charts of 115 patients were reviewed. Seventy-five patients $(65 \%)$ were treated empirically. Forty patients $(35 \%)$ were treated based on microbiological data that were available before the initiation of antibiotics. Patients who received nonempirical treatment for HAP had a significantly greater acuity of illness than the empirically treated group. Thirty-seven patients (49\%) who received empirical therapy were treated according to either ATS or hospital guidelines for HAP. The use of guideline-concordant antimicrobial therapy had no measurable effect on in-hospital mortality (eight of 37 patients [21.6\%] versus seven of 38 patients
[18.4\%], $\mathrm{P}=0.96)$ or median length of stay (19 days versus 21 days, $\mathrm{P}=0.30$ ). Patients whose treatment did not follow guideline recommendations tended to receive appropriate antimicrobial coverage more often than did those patients whose treatment was guideline concordant (15 of 18 patients [83\%] versus six of 11 patients [55\%], $\mathrm{P}=0.49$ ).

CONCLUSIONS: Institutional and ATS guidelines for the empirical treatment of HAP are less widely used than would be predicted by chance at The University Health Network. The clinical utility of these guidelines remains to be proven.

Key Words: Antibiotics; Guidelines; Nosocomial infection; Pneumonia

\section{Application des directives pour le traitement empirique de la pneumonie nosocomiale}

HISTORIQUE : Plusieurs séries de directives pratiques sur l'antibiothérapie empirique dans la pneumonie nosocomiale ont été mises au point, mais on ignore dans quelle mesure ces directives sont acceptées et utilisées.

OBJECTIF : Évaluer l'application des directives thérapeutiques empiriques de l'American Thoracic Society (ATS) et de notre établissement (The University Health Network) par les médecins dans les cas de pneumonie nosocomiale.

MODÈLE : Revue rétrospective des dossiers.

CONTEXTE : Hôpital universitaire.

voir page suivante 
PATIENTS : Cent quinze patients consécutifs ayant reçu un diagnostic de pneumonie plus de 48 heures après leur admission à 1'hôpital au cours d'une période de dix mois.

RÉSULTAT S : Les dossiers de 115 patients ont été passés en revue. Soixante-quinze patients $(65 \%)$ ont été traités de façon empirique. Quarante patients (35\%) ont été traités en fonctions des résultats d'analyses microbiologiques accessibles avant l'instauration des antibiotiques. Les patients qui ont reçu le traitement non empirique pour pneumonie nosocomiale présentaient une maladie beaucoup plus aiguë que les patients traités empiriquement. Trente-sept patients (49\%) ayant reçu le traitement empirique ont été traités conformément aux directives de l'ATS ou aux directives de l'hôpital pour la pneumonie nosocomiale. L'utilisation d'une antibiothérapie conforme aux direc- tives n'a exercé aucun effet mesurable sur la mortalité durant l'hospitalisation (8 patients sur 37 [21,6\%] versus 7 patients sur $38[18,4 \%$ ], $p=0,96)$ ni sur la durée médiane des séjours (19 jours versus 21 jours, $p=0,30)$. Les patients dont le traitement ne correspondait pas aux recommandations avaient tendance à recevoir une couverture d'antibiothérapie appropriée plus souvent que les patients dont le traitement était conforme aux directives (15 patients sur 18 [83\%], contre 6 patients sur 11 [55\%], $p=0,49)$.

CONCLUSION : Les directives de notre établissement de soins de santé et de l'ATS pour le traitement empirique de la pneumonie nosocomiale sont moins couramment utilisées qu'on aurait pu le prévoir dans notre établissement. L'utilité clinique de ces directives reste à démontrer.
$\mathrm{H}^{2}$ ospital-acquired pneumonia (HAP) occurs at a rate of five to 10 cases per one thousand hospital admissions, and its incidence is six to 20 times greater in patients receiving conventional mechanical ventilation (1). HAP is the leading cause of death due to nosocomial infection in the United States, with mortality rates as high as $40 \%$ despite antibiotic therapy (2-4). Previous work has shown that delays in the administration of appropriate antibiotic therapy are associated with increased mortality (5). The initiation of empirical antimicrobial therapy before the availability of diagnostic culture results has become a standard practice in the care of patients with HAP. Guidelines for the empirical treatment of HAP have been published by the American Thoracic Society (ATS) and are very similar to the Canadian guidelines published two years earlier $(6,7)$. In addition, many hospitals worldwide have developed institutional guidelines that reflect local practice patterns and microbial susceptibilities (8).

The majority of practice guidelines for the treatment of community-acquired pneumonia (CAP) or HAP have been developed by expert panels using consensus processes (9). The goals of practice guidelines are the standardization of patient care, cost containment and improvement in outcomes. While widespread implementation of many guidelines remains an ongoing challenge, a recent study done at our institution (The University Health Network, Toronto, Ontario) showed a high rate of adherence to treatment guidelines for CAP $(10,11)$. The primary objective of the present study was to assess the use of institutional and ATS guidelines for the empirical antimicrobial treatment of HAP at a tertiary care teaching hospital.

\section{PATIENTS AND METHODS}

The study setting was a two-campus, 1100-bed university teaching hospital (The Toronto General Hospital and the Toronto Western Hospital of the University Health Network). Data were collected in a retrospective fashion. The hospital's computerized patient database was searched using the International Classification of Diseases (ICD)-9 code for HAP to identify the inpatient records of all consecutive patients who received a diagnosis of pneumonia after admission to hospital between December 1995 and September 1996.

One of the authors reviewed each chart labelled with a diagnosis of HAP to verify that clear documentation of pneumonia had been made by the responsible house staff or attending physician. Those patients fulfilling a clinical diagnosis of pneumonia at $48 \mathrm{~h}$ or more after admission to hospital were defined as having HAP. Each chart was also searched for the presence of any of the following exclusion criteria, which were defined a priori: diagnosis of HAP made at an outside hospital; absence of treatment of HAP with antimicrobials; known positivity for human immunodeficiency virus (HIV) antibody; neutropenia (defined as an absolute neutrophil count of less than 1500 cells/mL; active tuberculosis; status postlung transplantation; and cystic fibrosis. Patients with HAP who had none of the exclusion criteria formed the study population. The inpatient records of these patients were then reviewed in detail to extract the relevant data.

Data assembly included age, sex, admitting diagnosis and admitting service, date of pneumonia diagnosis and location of the patient at the time of diagnosis of HAP (ward or intensive care unit). Vital signs and the use of supplemental oxygen, mechanical ventilation, vasopressor and/or inotropic agents and dialysis at the time of diagnosis were recorded. Patients receiving mechanical ventilation at the time of diagnosis were considered to have ventilator-associated pneumonia (VAP). A search was conducted for comorbidities including coma, head trauma, diabetes mellitus, renal failure (serum creatinine greater than 1.5 times the upper limit of normal), systemic steroid use, intravenous drug use, obstructive and/or structural lung disease, malignancy and a length of intensive care unit stay of more than five days before diagnosis of HAP. These comorbidities have been defined as risk factors for HAP by the ATS (6).

Patients were classified as having severe pneumonia according to the definition proposed by the ATS in its consensus statement on HAP (6). If the diagnosis of HAP was made while a patient was in an intensive care unit, the pneumonia was also classified as severe. The length of hospital stay and survival to discharge from hospital were recorded for each patient.

All available microbiological data were collected. Patients whose initial therapy was based on the available antimicrobial sensitivities of one or more presumed pulmonary pathogens formed the nonempirical treatment group. The remainder of the study population had no available microbiological data at the time of initiation of antibiotics for HAP, and those patients formed the empirical treatment group.

The antibiotic therapy administered to patients in the em- 
TABLE 1

Clinical characteristics of 115 patients with hospital-acquired pneumonia (HAP)

\begin{tabular}{|c|c|c|c|}
\hline Patient characteristics & Empirical treatment $(n=75)$ & Nonempirical treatment $(n=40)$ & $\mathbf{P}^{\star}$ \\
\hline Age (mean years \pm SD) & $63.3 \pm 14.1$ & $65.1 \pm 14.0$ & NS \\
\hline Female (\%) & $33(44.0)$ & $11(27.5)$ & NS \\
\hline Postoperative (\%) & $47(62.7)$ & $32(80.0)$ & NS \\
\hline Diagnosis in intensive care unit (\%) & $24(32.0)$ & $28(70.0)$ & 0.0002 \\
\hline Ventilator-associated pneumonia (\%) & $5(6.7)$ & $17(42.5)$ & 0.00001 \\
\hline Severe HAP (reference 6) (\%) & $33(44.0)$ & $30(75.0)$ & 0.003 \\
\hline More than one comorbidity ${ }^{\dagger}(\%)$ & $21(28.0)$ & $14(32.5)$ & NS \\
\hline Time to diagnosis (median days [range]) & $6.0(2$ to 180$)$ & 7.5 (2 to 62$)$ & NS \\
\hline In-hospital mortality (\%) & $15(20.0)$ & $6(15.0)$ & NS \\
\hline Length of hospital stay (median days [range]) & 20 (7 to 289$)$ & $32.5(10$ to 208$)$ & 0.003 \\
\hline
\end{tabular}

${ }^{*}$ Bonferroni correction; ${ }^{\dagger}$ Comorbidities include coma, head trauma, diabetes mellitus, renal failure (serum creatinine greater than 1.5 times the upper limit of normal), systemic corticosteroid use, intensive care unit stay longer than five days, intravenous drug use, obstructive and/or structural lung disease, and known malignancy. NS Not significant

pirical treatment group was recorded. It was then determined whether the agent (or agents) was prescribed in accordance with ATS guidelines, institutional guidelines, both or neither. The Canadian HAP guidelines (7) were not used for the purposes of the present study because of their similarity to the more recently published ATS guidelines.

The Antibiotic Subcommittee of the Pharmacy and Therapeutics Committee of the authors' institution has its own Guidelines for Antimicrobial Use (12), which are summarized in a handbook that is distributed to all house staff and medical staff at the beginning of each academic year. The guidelines for the empirical treatment of HAP outlined in this handbook are similar but not identical to those recommended by the ATS.

Categorical variables were compared using the ${ }^{2}$ test with continuity correction or Fisher's exact test when appropriate due to small sample size. Continuous variables were compared using the Mann-Whitney U test. All P values were two-tailed, and the $\mathrm{P}$ value for statistical significance was corrected by the Bonferroni method. Analyses were done using SAS 7.0 (SAS Institute Inc, USA).

\section{RESULTS}

The computerized search identified 169 inpatient charts. Fifty-four charts were excluded. The diagnosis of pneumonia was not considered by the responsible physician(s) in 20 patients. Of the remaining 34 patients, 14 had received a diagnosis of pneumonia less than $48 \mathrm{~h}$ after hospital admission, four were diagnosed at an outside hospital, three did not receive antimicrobial therapy, six were neutropenic at the time of diagnosis, two were HIV antibody-positive, one was receiving immunosuppressive therapy after lung transplantation and four charts were incomplete. Of the 115 patients eligible for the study, $75(65 \%)$ received empirical treatment for HAP and 40 (35\%) were treated based on microbiological data that were available at the time of initiation of antibiotics. The characteristics of these patients are shown in Table 1.

Patients treated nonempirically were more likely to be in the intensive care unit at the time of HAP diagnosis $(70.0 \%$
TABLE 2

Use of hospital-acquired pneumonia (HAP) guidelines in the empirical treatment group

\begin{tabular}{lcr}
\hline Location of admission & \multicolumn{1}{c}{$\begin{array}{c}\text { No HAP } \\
\text { guideline use }\end{array}$} \\
\hline Medical services $^{*}(\%)$ & 18 of $27(66.6)$ & 9 of $27(33.3)$ \\
Surgical services $(\%)$ & 19 of $48(39.6)$ & 29 of $48(60.4)$ \\
Total $(\%)$ & 37 of $75(49.3)$ & 38 of $75(50.7)$ \\
\hline
\end{tabular}

${ }^{*} P=0.04$ for the comparison of HAP guideline use between medical and surgical services

versus $32.0 \%, \mathrm{P}=0.0002$ ) and had a higher prevalence of $\operatorname{VAP}(42.5 \%$ versus $6.7 \%, \mathrm{P}=0.00001)$. The patients in this group had a significantly longer length of hospital stay (median 32.5 days versus 20.0 days, $\mathrm{P}=0.003$ ) but had an inhospital mortality rate similar to that of the patients in the empirical treatment group (15\% versus $20 \%$, not significant).

Antimicrobial therapy that was concordant with either the ATS or the hospital guidelines was used in 49\% (37 of 75) of patients treated empirically for HAP. Treatment was in accordance with the hospital guidelines in 36 of 37 patients, and with the ATS guidelines in 15 of 37 patients. The treatment of 14 of 15 patients who received therapy concordant with the ATS guidelines was also concordant with the hospital guidelines. Patients admitted to medical services were more likely to receive guideline-concordant therapy than were patients admitted to surgical services (67\% versus $40 \%$, $\mathrm{P}=0.04$ ) (Table 2).

Patients whose empirical antibiotic therapy did not follow a guideline were more likely to have severe HAP than were patients whose treatment was in accordance with either of the guidelines (58\% versus 30\%, not significant). In-hospital mortality was comparable in both groups (22\% versus $18 \%$, not significant), and there was no difference in the median length of hospital stay between the groups (21 days versus 19 days, not significant). Fewer antibiotics were used in patients whose treatment followed guideline recommendations (mean difference of 0.5 medications per patient, $\mathrm{P}<0.001$ ) (Table 3 ). 
TABLE 3

Characteristics of 75 patients with hospital-acquired pneumonia (HAP) who received empirical therapy

\begin{tabular}{|c|c|c|c|}
\hline Patient characteristics & Guideline use (n=37) & No guideline use $(n=38)$ & $\mathbf{P}^{*}$ \\
\hline Age (mean years $\pm S D)$ & $61.6 \pm 15.3$ & $64.8 \pm 12.9$ & NS \\
\hline Female (\%) & $18(48.7)$ & $15(39.5)$ & NS \\
\hline Postoperative (\%) & $20(54.1)$ & $27(71.1)$ & NS \\
\hline Diagnosis in intensive care unit (\%) & $7(19.0)$ & $17(44.7)$ & NS \\
\hline Ventilator-associated pneumonia (\%) & $2(5.4)$ & $3(7.9)$ & NS \\
\hline Severe HAP (reference 6) (\%) & $11(29.7)$ & $22(57.9)$ & NS \\
\hline More than one comorbidity ${ }^{\dagger}(\%)$ & $14(37.8)$ & $7(18.4)$ & NS \\
\hline Time to diagnosis (median days [range]) & 7 (2 to 180$)$ & 5 (2 to 140$)$ & NS \\
\hline $\begin{array}{l}\text { Number of antibiotics prescribed per patient for HAP } \\
(\text { mean } \pm \text { SD) }\end{array}$ & $1.2 \pm 0.6$ & $1.7 \pm 0.4$ & $<0.001$ \\
\hline Symptomatic improvement (\%) & $27(73.0)$ & $24(63.2)$ & NS \\
\hline In-hospital mortality (\%) & $8(21.6)$ & $7(18.4)$ & NS \\
\hline Length of hospital stay (median days [range]) & 19.0 (8 to 289$)$ & $21.0(7$ to 160$)$ & NS \\
\hline
\end{tabular}

${ }^{*}$ Bonferroni correction; ${ }^{\dagger}$ Comorbidities include coma, head trauma, diabetes mellitus, renal failure (serum creatinine greater than 1.5 times the upper limit of normal), systemic corticosteroid use, intensive care unit stay longer than five days, intravenous drug use, obstructive and/or structural lung disease, and known malignancy. NS Not significant

Microbiological data (culture and sensitivity results) from sputum specimens, bronchoscopic washings and/or blood cultures became available after the initiation of antibiotic treatment in $29(39 \%)$ of the empirically treated patients. Of the 11 patients treated in accordance with HAP guidelines, only six received the appropriate antimicrobial coverage compared with 15 of the 18 patients whose empirical therapy did not conform to either guideline (55\% versus $83 \%$, not significant). The first of the five patients receiving inappropriate coverage was a 68 -year-old woman who had undergone a thoracotomy for lung carcinoma five days previously. She had mild to moderate pneumonia by ATS criteria, and was treated with intravenous cefuroxime. Subsequent sputum culture revealed methicillin-sensitive Staphylococcus aureus. The patient had no known risk factors for staphylococcal pneumonia. The second patient was a 79-year-old woman with a history of chronic obstructive pulmonary disease who suffered acute renal failure after the repair of an abdominal aortic aneurysm. The pneumonia was classified as mild to moderate, and chest roentgenography revealed a pleural effusion. The patient was treated with intravenous cefuroxime. Culture of the pleural fluid grew Enterobacter aerogenes sensitive only to trimethoprim/sulfamethoxazole (TMP/SMX) and gentamicin. The third patient was a 60 -year-old man who was receiving mechanical ventilation two days after uncomplicated aortocoronary bypass surgery. He was initially treated with cefotaxime, which was concordant with the hospital guidelines but not with the ATS guidelines. Culture of the endotracheal aspirate revealed Enterobacter cloacae sensitive to TMP/SMX, gentamicin and ceftazidime. The fourth patient was diagnosed with HAP two days after undergoing cholangiojejunostomy for recurrent cholangiocarcinoma. He was initially treated with intravenous cefuroxime for mild to moderate HAP. Sputum culture revealed both methicillin-sensitive $S$ aureus and Serratia species, which were sensitive to TMP/SMX and gentamicin. The patient had no known risk factors for staphylococcal pneu- monia. The fifth patient was a 30-year-old woman who had previously undergone renal transplantation for end-stage renal disease secondary to type 1 diabetes mellitus. She was admitted with worsening renal function, and was diagnosed with mild to moderate HAP four days after admission. The patient received empirical treatment with intravenous cefuroxime, which was concurrent with the hospital guidelines but not with the ATS guidelines due to concomitant immunosuppressive therapy with corticosteroids, cyclosporine and azathioprine. Sputum culture subsequently revealed Pseudomonas aeruginosa, which was sensitive to fluoroquinolones, aminoglycosides, piperacillin and ceftazidime. None of the five patients died while in hospital.

Antibiotics not recommended by either guideline but which provided adequate antimicrobial coverage according to sensitivity data included ciprofloxacin, TMP/SMX, aminoglycosides, and the combination of vancomycin or erythromycin with second- or third-generation cephalosporins.

Overall, the most commonly prescribed empirical antibiotic was cefuroxime, a second-generation cephalosporin. Clindamycin was used in 13 patients, a third-generation cephalosporin in 11 patients, and ciprofloxacin was used alone or in combination with another antibiotic in $10 \mathrm{pa}-$ tients. TMP/SMX was used in five patients.

\section{DISCUSSION}

To our knowledge, this study is the first to assess the clinical use of practice guidelines for the empirical treatment of HAP. The data indicate that, unlike hospitalized patients with CAP, one-third of patients with HAP do not require empirical antimicrobial therapy because of the availability of microbiological data at the time of diagnosis (11). Almost one-half of all patients with severe HAP were treated nonempirically, suggesting that HAP treatment guidelines may actually have limited application in this subpopulation.

The overall rate of guideline compliance in the empirical treatment group was about 50\%. During the same period at 
our institution, a higher rate of guideline adherence was observed in the treatment of hospitalized patients with CAP (11). While medical services used guidelines to treat HAP to a significantly greater degree than did surgical services, the rate of guideline use by medical services remains lower than that observed in the aforementioned study (11) (67\% versus $81 \%$ ). Some physicians may have been ignorant of the existence of the guidelines. Other physicians may have elected not to use HAP guidelines due to the lack of prospective data supporting their use, the perceived nonapplicability of the guidelines in individual patients and pre-established practice patterns. Previous work has shown that although physicians may be aware of the existence of a practice guideline, actual knowledge of the guideline content is poor (13). There is no organized effort to ensure that physicians have access to the hospital guidelines for the treatment of HAP at our institution. However, even after the publication and widespread dissemination of guidelines on blood pressure management in the United States and smoking cessation in the United Kingdom, physician practice was found not to have changed $(14,15)$. Consequently, the mere existence of guidelines may not be effective in changing prescribing patterns. An alternative explanation for the discrepancy in the use of CAP and HAP guidelines is that the physicians taking part in the prospectively performed CAP study were more likely to comply with guidelines as a result of being aware that their prescribing habits were being monitored (10).

Although the group whose empirical therapy did not follow guideline recommendations was more severely ill, we did not observe a difference in mortality or in length of hospital stay relative to the guideline-concordant group. A recent prospective study that evaluated the use of ATS guidelines for the treatment of CAP also found no evidence of improved medical outcomes in treatment-concordant patients with one or more conditions (16). However, a subsequent study in elderly patients has reported a reduction in 30-day mortality with the use of therapy that is concordant with the ATS guidelines for CAP (17). In the empirical treatment group, the use of HAP treatment guidelines was associated with the prescription of fewer antibiotics per patient for pneumonia treatment, suggesting the potential for cost savings. Furthermore, the use of fewer antibiotics has been shown to decrease antimicrobial resistance among pulmonary pathogens $(18,19)$. However, analysis of the available microbiological data revealed that patients treated according to guidelines tended to receive less adequate antimicrobial coverage than did patients whose therapy did not match guideline recommendations. The use of therapy that was concordant with either the hospital or the ATS guidelines, or both, failed to include coverage for cephalosporin-resistant Enterobacter species, methicillin-sensitive $S$ aureus, $P$ aeruginosa and Serratia species in five patients. The use of therapy concordant with the ATS guidelines would have resulted in appropriate coverage in the two patients in this subgroup who received therapy concordant only with the hospital guidelines. The failure of Enterobacter species coverage due to cephalosporin resistance may be a result of the widespread use of second- and third-generation cephalosporins in our institution. Cefuroxime is recommended for mild to moderate HAP by both the ATS and the hospital guidelines, and was the most commonly prescribed antibiotic for HAP at our institution. Given that neither of the cases of $S$ aureus pneumonia had clinical risk factors for this organism, it is unlikely that coverage would have been any better if nonconcordant therapy had been rationally prescribed. However, previous work has confirmed that appropriate antibiotic coverage decreases mortality in patients with HAP (20,21). If these results are representative of what occurred in each group as a whole, the higher than expected mortality rate observed in the patients treated according to guidelines may be explained by the less adequate antimicrobial coverage in this group. In our study, physicians seemed to perform better than empirical guidelines in selecting appropriate empirical antibiotic coverage for HAP.

Despite the finding that the nonempirical group comprised a more sick subset of patients with a longer length of hospital stay, the in-hospital mortality rate was similar to that observed in the empirical group. This surprising result may be explained by the fact that patients in the nonempirical group received pathogen-directed therapy as opposed to empirical antimicrobial therapy. Furthermore, the greater severity of illness in these patients might have intensified the search for a responsible pathogen. Some investigators recommend bronchoscopy and bronchoalveolar lavage in all patients with VAP to avoid prescribing empirical antibiotic therapy, although this approach remains controversial (22-24).

The limitations of the present study are the small sample size and the retrospective design. It is possible that some cases of HAP were missed by the computerized search due to coding errors. On the basis of the experience of other groups, the observed mortality rates in this study were lower than expected (1). The chart review revealed that the treating physicians rarely documented the rationale for their antibiotic choices. The deliberate use or nonuse of guidelines for empirical therapy could, therefore, not be ascertained. The retrospective nature of the study poses significant barriers to making definitive conclusions about the determinants of physicians' prescribing practices in the management of HAP. The difficulty in ascertaining the morbidity and mortality attributable to HAP in patients with multiple comorbidities is a feature of retrospective and prospective studies. The generalizability of the study is limited by the fact that the study is the experience at a single centre. Despite these limitations, our results suggest that more data on patient outcomes, adequacy of antimicrobial coverage and pharmacoeconomic advantages are needed before there is wider physician acceptance of guidelines for the empirical treatment of HAP.

\section{CONCLUSIONS}

A substantial proportion of patients with HAP at our institution were treated based on available microbiological data. The use of guideline-recommended therapy for the empirical treatment of HAP is not more common than what would be 
predicted by chance. We believe that the results of this retrospective study mandate a large scale, prospective validation of published guidelines for the empirical antimicrobial therapy of HAP.

ACKNOWLEDGEMENTS: This study was approved by the Research Ethics Board of The University Health Network, Toronto, Ontario. No source of funding was used for this study.

\section{REFERENCES}

1. Celis R, Torres A, Gatell JM, Almeda M, Rodriguez-Roisin R, Agusti-Vidal A. Nosocomial pneumonia: a multivariate analysis of risk and prognosis. Chest 1988;93:318-24.

2. Hospitalizations for the leading causes of death among the elderly United States, 1987. MMWR Morb Mortal Wkly Rep 1990;39:777-9,785.

3. Fagon J-Y, Chastre J, Hance AJ, Montravers P, Novara A, Gibert C. Nosocomial pneumonia in ventilated patients: a cohort study evaluating attributable mortality and hospital stay. Am J Med 1993;94:281-8.

4. Chow CW, Lee-Pack LR, Senathiragah N, Rawji M, Chan M, Chan CK. Community acquired, nursing home acquired, and hospital acquired pneumonia: A five-year review of the clinical, bacteriological and radiological characteristics. Can J Infect Dis 1995;6:317-25.

5. Luna CM, Vujacich P, Niederman MS, et al. Impact of BAL data on the therapy and outcome of ventilator-associated pneumonia. Chest 1997;111:676-85.

6. American Thoracic Society. Hospital-acquired pneumonia in adults: diagnosis, assessment of severity, initial antimicrobial therapy, and preventative strategies. Am J Respir Crit Care Med 1996;153:1711-25.

7. Mandell LA, Marrie TJ, Niederman MS. Initial antimicrobial treatment of hospital acquired pneumonia in adults: A conference report. Can J Infect Dis 1993;4:317-21.

8. Mandell LA, Campbell GD Jr. Nosocomial pneumonia guidelines: an international perspective. Chest 1998;113(3 Suppl):188S-93S

9. Hackner D, Tu G, Weingarten S, Mohsenifar Z. Guidelines in pulmonary medicine. A 25-year profile. Chest 1999;116:1046-62.

10. Heffner JE. Does evidence-based medicine help the development of clinical practice guidelines? Chest 1998;113(3 Suppl):172S-8S.

11. Marras TK, Chan CK. Use of guidelines in treating community-acquired pneumonia. Chest 1998;113:1689-94.
12. The University Health Network. Guidelines for Antimicrobial Use. Toronto: The University Health Network, 1995.

13. Lomas J, Anderson GM, Domnick-Pierre K, Vayda E, Enkin MW, Hannah WJ. Do practice guidelines guide practice? The effect of a consensus statement on the practice of physicians. N Engl J Med 1989;321:1306-11

14. Hill MN, Levine DM, Whelton PK. Awareness, use, and impact of the 1984 Joint National Committee consensus report on high blood pressure. Am J Public Health 1988;78:1190-4.

15. Fowler G, Mant D, Fuller A, Jones L. The "Help Your Patient Stop" initiative: evaluation of smoking prevalence and dissemination of WHO/UICC guidelines in UK general practice. Lancet 1989;i:1253-5.

16. Gleason PP, Kapoor WN, Stone RA, et al. Medical outcomes and antimicrobial costs with the use of the American Thoracic Society guidelines for outpatients with community-acquired pneumonia. JAMA 1997;278:32-9.

17. Gleason PP, Meehan TP, Fine JM, Galusha DH, Fine MJ. Associations between initial antimicrobial therapy and medical outcomes for hospitalized elderly patients with pneumonia. Arch Intern Med 1999;159:2562-72

18. Rahal JJ, Urban C, Horn D, et al. Class restriction of cephalosporin use to control total cephalosporin resistance in nosocomial Klebsiella. JAMA 1998;280:1233-7.

19. Frank MO, Batteiger BE, Sorensen SJ, et al. Decrease in expenditures and selected nosocomial infections following implementation of an antimicrobial-prescribing improvement program. Clin Perform Qual Health Care 1997;5:180-8.

20. Torres A, Aznar R, Gatell JM, et al. Incidence, risk, and prognosis factors of nosocomial pneumonia in mechanically ventilated patients. Am Rev Respir Dis 1990;142:523-8.

21. Kollef MH, Ward S. The influence of mini-BAL cultures on patient outcomes: implications for the management of ventilator-associated pneumonia. Chest 1998;113:412-20.

22. Chastre J, Fagon J-Y. Invasive diagnostic testing should be routinely used to manage ventilated patients with suspected pneumonia. Am J Respir Crit Care Med 1994;150:570-4.

23. Niederman MS, Torres A, Summer W. Invasive diagnostic testing is not needed routinely to manage suspected ventilator-associated pneumonia. Am J Respir Crit Care Med 1994;150:565-9.

24. Sanchez-Nieto JM, Torres A, Garcia-Cordoba F, et al. Impact of invasive and noninvasive quantitative culture sampling on outcome of ventilator-associated pneumonia: a pilot study. Am J Respir Crit Care Med 1998;157:371-6. 


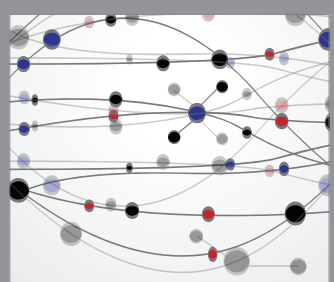

The Scientific World Journal
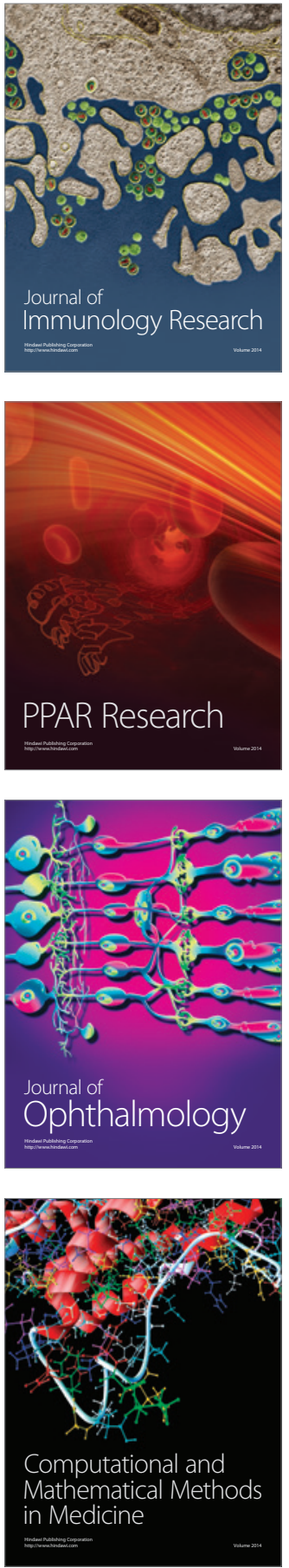

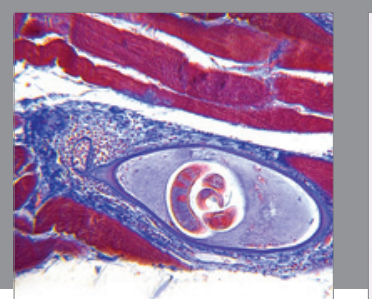

Gastroenterology Research and Practice

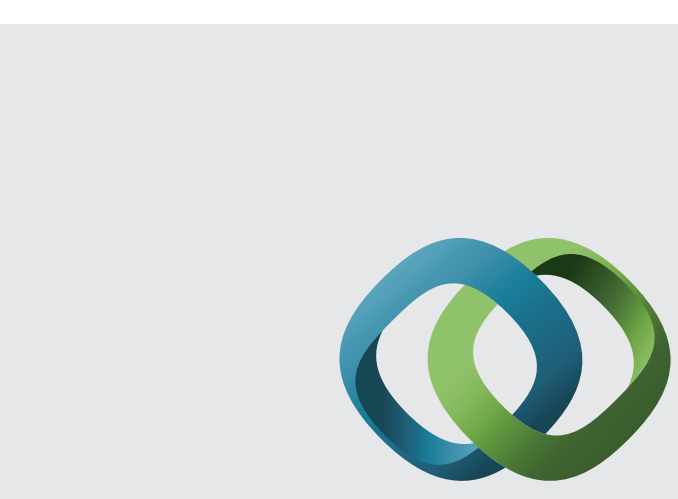

\section{Hindawi}

Submit your manuscripts at

http://www.hindawi.com
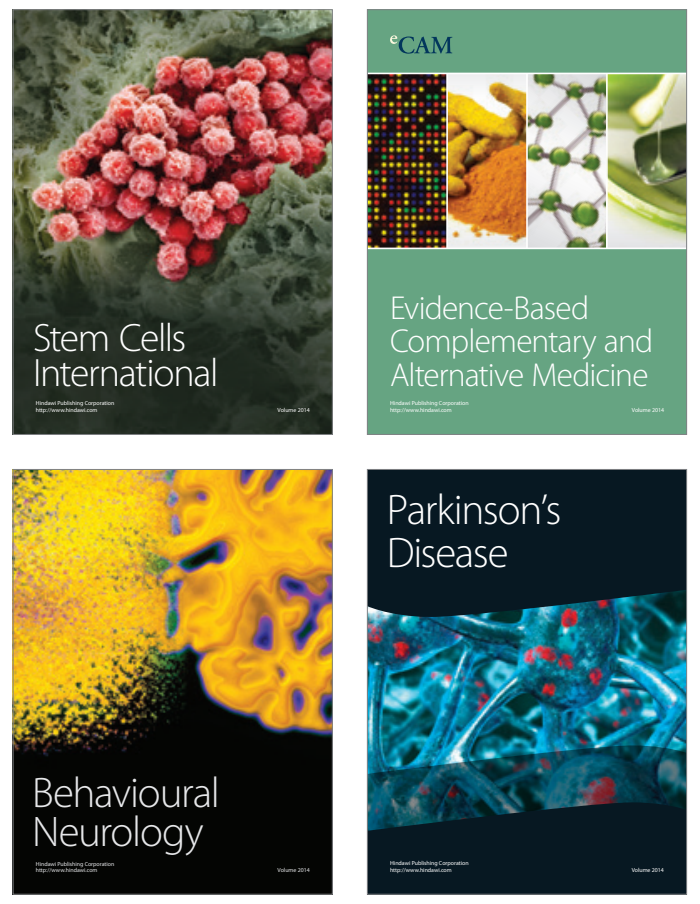
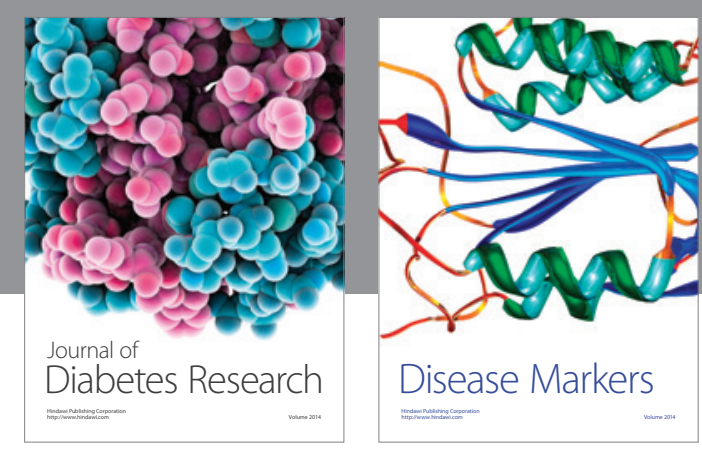

Disease Markers
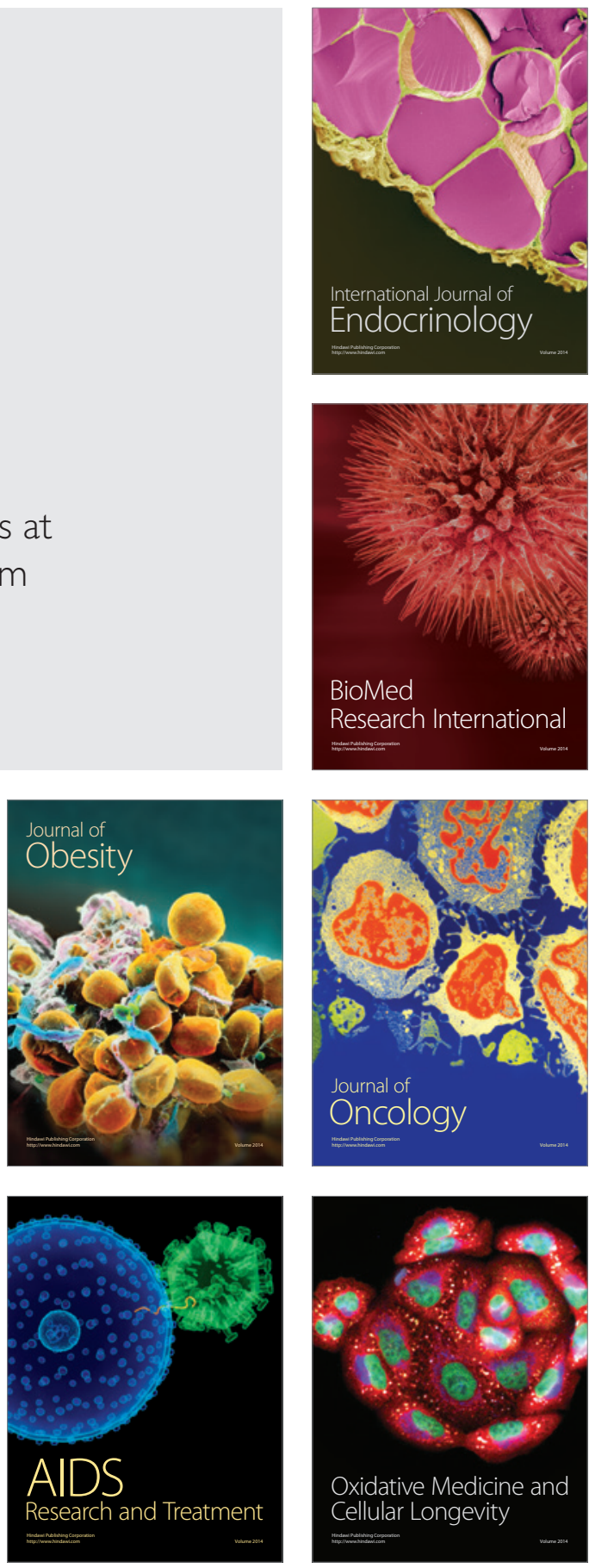